\title{
Non-Riemannian Geometry of Twisted Flux Tubes
}

\author{
L. C. Garcia de Andrade \\ Departamento de Física Teórica, Instituto de Física, UERJ, Brazil
}

\section{Received on 4 October, 2006}

\begin{abstract}
New examples of the theory recently proposed by Ricca [PRA(1991)] on the generalization of Da RiosBetchov intrinsic equations on curvature and torsion of classical non-Riemannian vortex higher-dimensional string are given. In particular we consider applications to 3-dimesional fluid dynamics, including the case of a twisted flux tube and the fluid rotation. In this case use is made of Da Rios equation to constrain the fluid. Integrals on the Cartan connection are shown to be related to the integrals which represent the total Frenet torsion and total curvature. By analogy with the blue phases twisted tubes in liquid crystals, non-Riemannian geometrical formulation of the twisted flux tube in fluid dynamics is obtained. A theorem by Ricca and Moffatt on invariant integrals for the Frenet curvature is used to place limits on the Cartan integrals. The stationary incompressible flow case is also addressed in the non-Riemannian case where Cartan torsion scalars are shown to correspond to abnormalities of the congruence. Geodesic motion is shown to be torsionless. Vorticity is shown to be expressed in terms of abnormalities of the congruence, which is analogous to the result recenly obtained [Garcia de Andrade,PRD(2004)], where the vorticity of the superfluid plays the role of Cartan contortion vector in the context of analog gravity.
\end{abstract}

Keywords: Twisted flux tubes; Non-Riemannanian geometry

\section{INTRODUCTION}

Differential geometry of curves and surfaces has appeared in several areas of physics, ranging from liquid crystals [1] to plasma physics [2], and from solitons [3] to general relativity [4], or even in high energy strings [5] and thermodynamics [6]. In all these applications one important common feature arises, which is the application of the Serret-Frenet frame to the motion of curves. Da Rios equation [7] on the other hand derived equations of motion of filaments based on the Frenet frame. These equations constraints the motion of curves, for example, in magnetohydrostatic [8] problems of nested toroidal flux surfaces. More recently, Rogers and Schief [3] have demonstrated that in steady hydrodynamic, geodesic motions constrained the curvature and torsion of the sreamlines necessarily implying the existence of travelling wave solutions of Da Rios equations. Da Rios equation have been recently revisited by Ricca [9]. Yet more recently, Garcia de Andrade [10] has applied another Ricca's paper [11] result on twisted magnetic flux tubes to electron fluids, followed by some work [11] on Riemannian geometry of vortex filaments in plasma physics. Ricca has also [12] generalized the Da Rios-Betchov equation to higher dimensional vortex string. Also recently Garcia de Andrade [13] has shown that it is possible to apply non-Riemannian geometry to vortex acoustics, to solve some problems with vorticity fluids in the analogue models for general relativity. Non-holonomic geometry in fluid mechanics has been first appeared in the literature on a paper of 1983 by Littlejohn paper [3] on the geometrical formulation of plasmas based on the Serret-Frenet frame, where use was made of asymmetric connection. This very same Cartan torsion is used here in the form of torsion scalars, which we apply to another example in fluid dynamics, which is a vortex tube. Another interesting way of computing the Cartan torsion in the flux tube is through teleparallelism, which is a version of non-Riemannian geometry where the to- tal, so-called Riemann-Cartan curvature, vanishes. This can be obtained by a special choice of Cartan connection form. Another way of computing the non-Riemannian geometry of the twisted tubes is by considering the case where the connection one-form does not vanish. The paper is organised as follows: Section 2 presents the fundamental form of the Serret-Frenet equation based on the Gauss equations. Section 3 presents the non-Riemannian geometry of twisted flux tubes along the teleparallel geometry. In section 4 the example of Cartan torsion scalars in stationary incompressible fluids is given. In section 5 solitonic Heisenberg spin equations are used to constraints magnetic fluids equations. Discussions and conclusions are presented in section 6 .

\section{NON-RIEMANNIAN GEOMETRY FROM GAUSS EQUATIONS IN SERRET-FRENET FRAME}

We start by writing the Serret-Frenet frame, composed of the vector triad $X=(\vec{t}, \vec{n}, \vec{b})$ in the more compact matrix form by

$$
\frac{\partial}{\partial s} X^{T}=A X^{T}
$$

where A corresponds to the following array

$$
\left(\begin{array}{ccc}
0 & \kappa & 0 \\
-\kappa & 0 & \tau \\
0 & -\tau & 0
\end{array}\right)
$$

while the other equations for $\vec{n}$ and $\vec{b}$ direction are given

$$
\begin{aligned}
\frac{\partial}{\partial n} X^{T} & =B X^{T} \\
\frac{\partial}{\partial b} X^{T} & =C X^{T}
\end{aligned}
$$


where $\mathrm{T}$ represents the transpose of the line matrix $\mathrm{X}$ and $\mathrm{B}$ and $\mathrm{C}$ are the respective skew-symmetric matrices

$$
\left(\begin{array}{ccc}
0 & \theta_{n s} & \Omega_{b}+\tau \\
-\theta_{n s} & 0 & -\operatorname{div} \vec{b} \\
-\left(\Omega_{b}+\tau\right) & \operatorname{div} \vec{b} & 0
\end{array}\right)
$$

and

$$
\left(\begin{array}{ccc}
0 & -\left(\Omega_{n}+\tau\right) & \theta_{b s} \\
\left(\Omega_{n}+\tau\right) & 0 & \kappa+\operatorname{div} \vec{n} \\
-\theta_{b s} & -(\kappa+\operatorname{div} \vec{n}) & 0
\end{array}\right)
$$

where $\theta_{n s}$ and $\theta_{b s}$ are respectively

$$
\theta_{n s}=\vec{n} \cdot \frac{\partial}{\partial n} \vec{t}
$$

and

$$
\theta_{b s}=\vec{b} \cdot \frac{\partial}{\partial b} \vec{t}
$$

The gradient operator is

$$
\nabla=\vec{t} \frac{\partial}{\partial s}+\vec{n} \frac{\partial}{\partial n}+\vec{b} \frac{\partial}{\partial b}
$$

The other vector analysis formulas read [14]

$$
\begin{gathered}
\operatorname{div} \vec{t}=\theta_{n s}+\theta_{b s} \\
\operatorname{div} \vec{n}=-\kappa+\vec{b} \cdot \frac{\partial}{\partial b} \vec{n} \\
\operatorname{div} \vec{b}=-\vec{b} \cdot \frac{\partial}{\partial n} \vec{n} \\
\nabla \times \vec{t}=\Omega_{s} \vec{t}+\kappa \vec{b}
\end{gathered}
$$

Similarly the results for $\vec{n}$ and $\vec{b}$ are given by

$$
\begin{gathered}
\nabla \times \vec{n}=-(\operatorname{div} \vec{b}) \vec{t}+\Omega_{n} \vec{n}+\theta_{n s} \vec{b} \\
\Omega_{n}=\vec{n} . \nabla \times \vec{n}=\vec{t} \cdot \frac{\partial}{\partial b} \vec{n}-\tau
\end{gathered}
$$

and

$$
\begin{aligned}
\nabla \times \vec{b} & =(\kappa+\operatorname{div} \vec{n}) \vec{t}-\theta_{b s} \vec{n}+\Omega_{b} \vec{b} \\
\Omega_{b} & =\vec{b} \cdot \nabla \times \vec{b}=-\vec{t} \cdot \frac{\partial}{\partial n} \vec{b}-\tau
\end{aligned}
$$

where

$$
\Omega_{s}=\vec{b} \cdot \frac{\partial}{\partial n} \vec{t}-\vec{n} \cdot \frac{\partial}{\partial b} \vec{t}
$$

which is called abnormality of the $\vec{t}-$ field. Most of these formulas were derived in Marris and Passman [14]. In what follows we apply some of those formulas to vortex and plasma filaments from the point of view of non-Riemannian geometry. Let us now consider Littlejohn idea of considering the expression

$$
\Gamma_{j k}^{i}=[\vec{t} . \nabla \times \vec{t}]_{j k}^{i}
$$

or

$$
\vec{t} . \nabla \times \vec{t}=\varepsilon^{i j k} t_{i} \partial_{j} t_{k}=\varepsilon^{i j k} \Gamma_{j k}^{i}
$$

as definitions of asymmetric affine connections. Here $\varepsilon^{i j k}$ is the totally skew Levi-Civita symbol and $t_{i}$ represents the covariant components of vector $\vec{t}$, while $\partial_{j}:=\frac{\partial}{\partial x^{j}}$ is the usual partial derivative operator in general coordinates $x^{j}$. Note that expression (17) defines a Cartan scalar due to the total skewsymmetric part of Levi-Civita symbol which kills the symmetric part of affine connection. From expression (10) the torsion scalar is easily computed as

$$
\vec{t} . \nabla \times \vec{t}=\Omega_{s}
$$

Thus the Cartan torsion scalar is equivalent to the abnormality of the $\vec{t}$-field. Let us now consider the relation between the Frenet and Cartan scalar torsions. The frame $\vec{X}:=\vec{e}_{i}$ here represents the Frenet frame. This allows us to write down the expressions for the Gauss equations of a surface (see $p .18$ of second reference in [3]) in the form

$$
\frac{\partial \vec{e}_{i}}{\partial x^{j}}=\Gamma_{i j}^{* l} \vec{e}_{l}
$$

where

$$
\frac{d}{d s} \vec{e}_{i}=\partial_{k} \vec{e}_{i} \frac{d x^{k}}{d s}
$$

It is clear that use has been made of the vanishing of the covariant derivative of the frame so that

$$
\frac{D \vec{e}_{i}}{D s}=\nabla_{j} \vec{e}_{i} \frac{d x^{j}}{d s}
$$

where $\frac{D}{D s}$ is the absolute derivative operator while $\nabla_{j}$ represents the covariant derivative operator and $\nabla_{j} \vec{e}_{i}:=\nabla_{\vec{e}_{j}} \vec{e}_{i}$. From equation (21) we obtain

$$
\varepsilon^{p k i} \vec{e}_{p} \cdot \nabla_{k} \vec{e}_{i}=\varepsilon^{p k i} \Gamma_{i k}^{l} \vec{e}_{p} \cdot \vec{e}_{l}=\varepsilon^{p k i} \Gamma_{i k}^{l} g_{p l}
$$

where the dot represents the scalar product of vetors and $\varepsilon^{i j k}$ is the Levi-Civita totally skew-symmetric symbol and covariant and partial derivatives of the frame $\vec{e} \vec{e}^{i}$ are proportional to the full non-Riemannian connection $\Gamma^{i}{ }_{j k}$. The expression $\frac{D \vec{e}_{i}}{D s}=0$ is very commom in the psedo-Riemannian Lorentz geometry of Einstein's general relativity ,representing the Fermi transport of particles along curves. Note also that here the metric $g\left(\vec{e}_{l}, \vec{e}_{k}\right)$ does not coincide with the Krönecker delta $\delta_{l k}$ unless the frame is orthogonal. By expressing the left-hand side of (22) as

$$
\left(\varepsilon^{p k i} \nabla_{k} \vec{e}_{i}\right) \cdot \vec{e}_{p}=\varepsilon^{p k i} \Gamma_{i k}^{l} g_{p l}
$$


In vector notation

$$
\varepsilon^{p k i} \nabla_{k} \vec{e}_{i}=\nabla \times \vec{X}
$$

where we have used the definition $\vec{X}=\vec{e}_{i}$. Yet in vectorial notation equation (22) finally reads

$$
\vec{X} . \nabla \times \vec{X}=\varepsilon^{p k i} \Gamma_{[i k]}^{l} g_{l p}
$$

where $g_{l p}$ is the manifold metric. Here $\Gamma_{[i k]}^{l}$ is Cartan connection, obtained from the skew-symmetric affine connection

$$
\Gamma_{i k}^{l}=\Gamma^{* l}{ }_{i k}-K_{i k}^{l}
$$

$\Gamma^{*}{ }_{i k}$ is the Christoffel symmetric connection and $K^{l}{ }_{i k}$ represents the Elie Cartan contortion [15]. The Cartan connection in elastic materials represents the defects in crystals [16] for example. In the case we consider that $\vec{X}$ is the Frenet-Serret frame the LHS of (25) are the abnormalities $\Omega_{\vec{X}}$ defined in equations (13) and (15). This can be easily seen from equations (16) and (17). Therefore we have shown that the torsion scalars coincide with the abnormalities of the congruence. Note that in the case of geodesic congruence $\Omega_{s}=0$ the torsion scalar vanishes, as happens in the torsionless Riemannian geometry. From the expressions (20) and (21) one obtains

$$
\Gamma_{k s}^{n} \frac{d x^{k}}{d s}=\kappa
$$

which yields the integral

$$
I_{1}=\int \Gamma_{k s}^{n} d x^{k}=\int \kappa d s
$$

This expression shows that the Frenet curvature is linked with the contortion. The integral on the RHS of (28) is called total Frenet curvature. The other integrals for $i=s$ and $i=b$ yields

$$
\begin{gathered}
I_{2}=\int \Gamma_{k n}^{s} d x^{k}=-\int \kappa d s \\
I_{3}=\int \Gamma^{b}{ }_{k n} d x^{k}=\int \tau d s
\end{gathered}
$$

where the last integral is the total Frenet torsion. In the following sections we notice that similar torsion integrals appear again. The Cartan torsion invariants discussed in this section can be used together with two theorems, one by Fukumoto (1987) [17] and its subsequent generalization by Moffatt and Ricca (1991) [18] to provide us with a generalization of their contents to Cartan torsion. See also reference [19] for a concise discussion and presentation of both theorems. Moffatt-Ricca theorem states that for a knotted filament, the total Frenet curvature is bounded above and below by

$$
4 \pi<\int \kappa d s<\text { constant }
$$

Therefore by expressions (29) and (30) we could may that these same bounds are valid for total Cartan torsion.

\section{TELEPARALLEL TWISTED FLUX TUBES}

Let us now very briefly consider the twisted topology and the metric of flux tubes represented by $\mathcal{T}:=S \times \mathcal{C}$, where $\mathcal{S}$ is the circular cross section of area $A=\pi R^{2}$, R being the radius of the circular section which is by construction small with respect to the radius of curvature $\mathrm{R}$ of the tube axis curve $\mathcal{C}$. The orthogonal curvilinear coordinates $\left(r, \theta_{R}, s\right)$ are centered on $\mathcal{C}$. Points on $\mathcal{S}$ are given by the vector

$$
\vec{x}=\vec{X}(s)+r \cos \theta(s) \vec{n}(s)+r \sin \theta(s) \vec{b}(s)
$$

where $\theta(s)$ is the polar angle refereed to the unit normal vector $\vec{n}$ in the Frenet frame composed by the $\operatorname{triad}(\vec{t}, \vec{n}, \vec{b})$, where $\vec{t}=\frac{d \vec{x}}{d s}$ is directed along to the curve $\mathrm{C}$ and $\vec{n}$ and $\vec{b}$ are the normal and binormal to the tube axis C. Angle $\theta(s)$ varies according to the tube geometry. Polar angle $\theta_{R}$ is an independent coordinate obeying the following relation

$$
\theta(s)=\theta_{R}+\gamma(s)
$$

$\gamma(s)$ is responsible for the contribution from Frenet torsion $\tau(s)$ of $\mathrm{C}$ is

$$
\gamma(s)=-\int \tau(\varepsilon) d \varepsilon
$$

where the integral ranges from 0 to $s$. The metric is

$$
d \vec{x} . d \vec{x}=d r^{2}+r^{2}\left(d \theta_{R}\right)^{2}+K^{2} d s^{2}
$$

which is orthogonal. The factor $\mathrm{K}(\mathrm{s})$ is

$$
K(s)=1-r \kappa(s) \cos \theta(s)(s)
$$

In Ricca's geometrical model for the twisted flux tube the orthogonal basis $\left(\vec{e}_{r}, \vec{e}_{\theta}, \vec{t}\right)$ is expressed as

$$
\begin{gathered}
\vec{e}_{r}=\vec{n} \cos \theta+\vec{b} \sin \theta \\
\vec{e}_{\theta}=-\vec{n} \sin \theta+\cos \theta \vec{b}
\end{gathered}
$$

The tubes here are twisted with the magnetic field lines winding about the magnetic flux-tube along the longitudinal direction $\vec{t}$. The total twist of the tube is

$$
T \omega=\frac{1}{2 \pi} \oint \tau d s+\frac{1}{2 \pi}\left[\theta^{*}\right]_{C}
$$

By making use of the Cartan calculus of differential forms we are able to expressed the twisted flux tube metric as

$$
d l^{2}=\left(\omega^{r}\right)^{2}+\left(\omega^{\theta}\right)^{2}+\left(\omega^{s}\right)^{2}
$$

where the basis one-forms are

$$
\begin{gathered}
\omega^{r}=d r \\
\omega^{\theta}=r d \theta
\end{gathered}
$$




$$
\omega^{s}=K(s) d s
$$

From the Poincare lemma: $d^{2}=0$ for the differential operator d, we obtain

$$
\begin{gathered}
d \omega^{r}=0 \\
d \omega^{\theta}=d r \wedge d \theta
\end{gathered}
$$

$$
d \omega^{s}=-\kappa(s) \cos (\theta+\gamma) d r \wedge d s+r \kappa(s) \sin (\theta+\gamma) d \theta \wedge d s
$$

where $\wedge$ is the exterior product sign between differential forms. From the torsion Cartan equation

$$
T^{i}=d \omega^{i}+\omega_{k}^{i} \wedge \omega^{k}
$$

where $\omega_{k}^{i}$ is the connection one-form and $T^{i}$ is the torsion two-form and $T^{i}=T^{i}{ }_{j k} \omega^{j} \wedge \omega^{k}$, where $T^{i}{ }_{j k}$ are the components of the Cartan torsion tensor. To simplify matters in this section we shall consider the special orthonormal frame where the connection form vanishes and from the Cartan curvature equation

$$
R_{j}^{i}:=\frac{1}{2} R_{j k l}^{i} \omega^{k} \wedge \omega^{l}=d \omega_{j}^{i}+\omega_{k}^{i} \wedge \omega_{j}^{k}
$$

which implies that the Riemann-Cartan curvature two-forms $R_{j}^{i}$ vanishes, which is the teleparallel condition. The torsion forms are easily computed as

$$
\begin{gathered}
T^{r}=0 \\
T_{r \theta}^{\theta}=\frac{1}{r} \\
T^{s}{ }_{\theta s}=\frac{\kappa}{K}\left[\theta-\int \tau d s\right]
\end{gathered}
$$

Here we have used the approximation of small torsion which yields

$$
\sin \left[\theta-\int \tau d s\right]=\theta-\int \tau d s
$$

One notes from expression (51), that the Cartan torsion component is proportional to the total torsion integral.

\section{NON-RIEMANNIAN GEOMETRY OF VORTEX FILAMENTS UNDER DA RIOS CONSTRAINTS}

In this section we show that torsion scalars or abnormalities, and Da Rios system play a fundamental role in the solution of stationary fluid equations by finding a solution where both Frenet curvature and torsion are not constants. The vortex filament has the following velocity equation

$$
\vec{v}=\kappa \vec{b}
$$

where the vortex filament rotation is given by

$$
\nabla \times \vec{v}=\vec{\Omega}
$$

Here

$$
\nabla=\vec{t} \frac{\partial}{\partial s}+\frac{\vec{b}}{\kappa} \frac{\partial}{\partial b}
$$

where $\nabla$ is the gradient operator. To investigate the relation between this hydrodynamics and torsion scalars, or abnormalities, we compute the term

$$
\vec{v} \cdot \nabla \times \vec{v}=\vec{v} \cdot \vec{\Omega}
$$

The first term on the LHS of this equation yields

$$
\vec{v} . \nabla \times \vec{v}=\kappa^{2} \Omega_{b}
$$

Comparison between the last two equations yields

$$
\vec{b} \cdot \vec{\Omega}=\kappa \Omega_{b}
$$

which clearly shows that the torsion scalar or abnormality $\Omega_{b}$ is proportional to rotation of the vortex tube. Before we compute explicitly the vorticity expression, we consider the incompressibility of the fluid

$$
\nabla \cdot \vec{v}=0
$$

Considering in principle that $\kappa=\kappa(s, b)$ we obtain

$$
\nabla \cdot \vec{v}=\frac{\kappa_{b}}{\kappa}=0
$$

shows that the Frenet curvature does not depend on the variable $\mathrm{b}$ and can be expressed in the form by $\kappa(s)$. Let us now compute the vorticity $\vec{\Omega}$ as

$$
\vec{\Omega}=\left[\left(\vec{t} \frac{\partial}{\partial s}+\frac{\vec{b}}{\kappa} \frac{\partial}{\partial b}\right) \times \kappa \vec{b}\right]=\kappa(\kappa+\operatorname{div} \vec{n}) \vec{t}+\kappa_{s} \vec{n}+\kappa \tau \vec{b}
$$

On the other hand $\vec{\Omega}$ can also be written as

$$
\vec{\Omega}=\nabla \times(\kappa \vec{b})=\nabla \kappa \times \vec{b}+\kappa \nabla \times \vec{b}
$$

Scalarly multiplying both sides of the equation (62) by the filament velocity $\vec{v}=\kappa \vec{b}$ and making use of expression (14) above yields

$$
\Omega_{b}=\frac{\vec{b} \cdot \Omega}{\kappa}
$$

From expressions (61) and (63) we obtain $\Omega_{b}=\tau$, which shows once more that abnormality, or Cartan torsion scalar, is proportional to the vortex filament Frenet torsion. Let us now consider Da Rios equation

$$
\begin{gathered}
\kappa_{b}=-2 \tau \kappa_{s}-\kappa \tau_{s} \\
\tau_{b}=\left[-\tau^{2}+\frac{1}{\kappa} \kappa_{s s}+\frac{1}{2} \kappa^{2}\right]_{s}
\end{gathered}
$$


Since $\kappa_{b}=0$, the Da Rios system reduce to

$$
2 \tau \kappa_{s}=-\kappa \tau_{s}
$$

and

$$
\left[-\tau^{2}+\frac{1}{\kappa} \kappa_{s s}+\frac{1}{2} \kappa^{2}\right]=c
$$

Since in the case of helical flows torsion is proportional to curvature, we considered $\tau_{b}=0$ to simplify matters. Here $c$ is an integration constant. Da Rios equation (67) yields a relation between torsion and Frenet curvature given by $\kappa^{2}=$ $\tau$. Substitution of this expression into the remaining Da Rios equation (67) yields

$$
\kappa_{s s}-c \kappa+\frac{1}{2} \kappa^{3}-\kappa^{5}=0
$$

which is a solitonic-like equation. This equation can be easily solved if we kept only terms up to first order on Frenet curvature. Integration of this equations yields

$$
\kappa=\kappa_{0} \exp (-\sqrt{c} s)
$$

which by the relation between $\tau$ and $\kappa$ yields

$$
\tau=\kappa_{0}^{2} \exp (-2 \sqrt{c s})
$$

From the expression $\Omega_{b}=\tau$ one may now compute the relation between vorticity and speed of the flow as

$$
\tau \kappa^{2}=\vec{v} \cdot \vec{\Omega}
$$

Using the relation obtained from Da Rios equation between torsion and curvature into (71) yields

$$
\tau^{2}=\vec{v} \cdot \vec{\Omega}
$$

This relation leads us to conclude that, for planar curves, where $\tau$ vanishes, the vorticity is orthogonal to the direction of fluid flow. Besides, substitution of expression (69) into (70) yields

$$
\vec{v} \cdot \vec{\Omega}=\kappa^{4}{ }_{0} \exp (-4 \sqrt{c} s)
$$

which shows that as $s \rightarrow \infty$, even for nonplanar curves, the effect of orthogonality betwen the vorticity and the flow direction cannot be recovered, unless we are very far from the center of the filament. Therefore the investigation of nonplanar curves in plasmas seems to be very of utmost importance for example, to the electron drift in solar loops, as has been recently demonstrated by the Garcia de Andrade [20].

\section{HEISENBERG SPIN EQUATIONS CONSTRAINTS IN MAGNETIC FLUIDS}

In this section we shall address the demonstration of the following simple theorem: THEOREM: The Heisenberg spin equations

$$
\vec{t}_{b}=\vec{t} \times \vec{t}_{s s}
$$

and Da Rios equations, allow us to show that the magnetic field obeying the conserved current along the b-line

$$
\overrightarrow{J_{b}}=0
$$

implies that momentum along the n-line $\Omega_{n}=-\tau$, as long as the form of the field is $\vec{B}=B(s) \vec{t}$. The absence of magnetic monopoles along the filaments imply that the modulus of the magnetic field is constant along the filament. This shows once more that the abnormality in direction- $n$ is proportional to Frenet torsion and consequently to non-Riemannian torsion in the flow. Proof: Let us first consider the Ampere law in the form

$$
\vec{J}=\nabla \times \vec{B}=B\left(\frac{\Omega_{n}+\tau}{\kappa} \vec{t}+\kappa \vec{b}\right)
$$

taking the partial derivative of this plasma current along the b-line yields

$$
\vec{J}_{b}=B\left[\frac{\Omega_{n}+\tau}{\kappa} \vec{t}_{b}+\kappa_{b} \vec{b}\right]
$$

which by making use of Heisenberg equation reduces to

$$
\overrightarrow{J_{b}}=B\left[\left[\frac{\Omega_{n}+\tau}{\kappa}\right] \vec{t} \times \vec{t}_{s s}+\kappa_{b} \vec{b}\right]
$$

Finally the absence of magnetic monopoles along the filament implies

$$
\nabla \cdot \vec{B}=\left(\vec{t} \frac{\partial}{\partial s}+\frac{\vec{b}}{\kappa} \frac{\partial}{\partial b}\right) \cdot(B \vec{t})=0
$$

After some algebra this yields $\nabla \cdot \vec{B}=B_{s}=0$ proves the last part of the theorem. In the second equality on the RHS of the equation (78) we used the solitonic Heisenberg spin equations.

\section{CONCLUSIONS}

We have investigate a simple connection between the Serret-Frenet and Gauss equations which naturally leads to the non-Riemannian formulation in 3D manifold and applied to fluid flows. Several examples of the application of this idea are presented and its connection to soliton surfaces hierarchy is discussed. Despite of the great success Riemannian geometry had in describing fluid motion [21] it seems interesting to investigate the role of non-Riemannian geometry in fluid dynamics as done here. Physical interpretation of contortion here is analogous to the interpretation it has in gravitation and electrodynamics in non-Riemannian spacetime recently discussed by Hehl and Obukhov [22] where spiral staircases are used. This would be analogous to the twisted tubes considered in this paper. Also here, the relation between the Cartan and Frenet torsions reminds the Cartan idea [15] where torsion was connected to the translation and rotation of space. Note that the analogy between the vorticity in superfluids [13] and the Cartan contortion can be used as a further motivation to investigate the role of Cartan torsion on the relation between Frenet and Cartan torsions in the dynamics of fluids. 
Besides in defect theory and even in gravitation, theorie such as Einstein-Cartan like the contortion are also called defect torsion. Though some material in this paper is a revision of previous work, the material on teleparallel geometry of tubes, theorem for Cartan torsion integral invariants compared with theorems by Fukumoto, Moffatt and Ricca and soliton equation for Frenet curvature seems to be new.

\section{Acknowledgements}

I would like to thank CNPq (Brazil) and Universidade do Estado do Rio de Janeiro for financial supports.
[1] E. Dubois Viollet, Which Universe for Blue Phase?, in Geometry in Condensed Matther Physics, Ed. J.F. Sadoc, World Scientific (1990).

[2] R. Littlejohn, Phys. Plasmas, 28, 2015 (1985).

[3] W.K. Schief and C. Rogers, The Da Rios system under a geometrical constraint: the Gilbarg problem, J. Geometry and Physics, v. 54, issue 3, 286-300, (2005). C. Rogers and Schief, Bäcklund Darboux transformations: Geometry and Modern Applications of Soliton theory, Cambridge University Press (2002) and references therein.

[4] V. de Sabbata and C. Sivaram, Spin and Torsion in Gravitation, World Scientific (1995).

[5] B. M. Barbashov, V. V. Nesterenko, Introduction to Relativistic String Theory, World Scientific (1990).

[6] R. Gilmore, Phys. Rev. A 30, 1994 (1984); G. Kuppeiner, Phys. Rev. A 24, 488 (1981)

[7] L. S. Da Rios, Rend. Circ. Mat. di Palermo 22, 117 (1906).

[8] W. Schief, Phys. Plasmas, 10, 2677 (2003).

[9] R. L. Ricca, Nature 352, 561 (1991). R. L. Ricca, Fluid Dynamics Research Journal, 36, 319 (2005).

[10] L. C. Garcia de Andrade, Physics of Plasmas, Feb issue online and Vortex Filaments in MHD, Phys. Scr. (2006) in press.

[11] R. Ricca, Journal of Fluid Mechanics, 237, 241 (1994).

[12] R. L. Ricca, Phys. Rev. A 43, 4281 (1991).
[13] L. C. Garcia de Andrade, Phys. Rev. D 70, 064004 (2004).

[14] A. W. Marris and S. L. Passmann, Arch. Rat. Mech. Analysis 32, 29 (1969).

[15] E. Cartan, Riemannian Geometry on Orthogonal Frames, World Scientific (2001).

[16] M. O. Katanaev and V. I. Volovich, Annals of Physics, 216, 1 (1992).

[17] Y. Fukumoto, On integral invariants for vortex motion under LIA, J. Phys. Soc. Japan, 56, 4207 (1987).

[18] K. H. Moffatt and R. Ricca, Interpretation of invariants of thr Betchov-Da Rios equations and of the Euler equations, in The Global Geometry of Turbulence, J. Jimenez, ed., 257-264, Plenum Press, New York (1991).

[19] P. K. Newton, The N-Vortex Problem: Analytical Techniques, Springer-Verlag (2001).

[20] L. C. Garcia de Andrade, Curvature and torsion effects in current-carrying twisted nonplanar solar loops, DFT-UERJpreprints-2006.

[21] T. Kambe, Geometrical theory of Dynamical Systems and Fluid Flows, World Scientific (2004).

[22] F. W. Hehl and Y. N. Obukhov, Foundations of Classical Electrodynamics: Charge, Flux, and Metric, Birkhä user (2003). 